\title{
Impact of Depression on Diabetes mellitus
}

\author{
Hermann Toplak $^{\mathrm{a}}$ Heidemarie Abrahamian ${ }^{\mathrm{b}}$ \\ a Department of Medicine, Medical University Graz, \\ b 3 rd Medical Department, General Hospital Hietzing, with Neurological Center Rosenhuegel and Karl Landsteiner Institute for Metabolic \\ Diseases and Nephrology, Vienna, Austria
}

The consideration of the interaction of psychological factors like distress and depression with human behavior has led to a better understanding of pathophysiology and treatment of chronic diseases. Stress factors associated with distress and depression relate to a loss of treatment adherence and compliance with consecutive rise in addictive behavior and accompanying complications [1], thus increasing the need of complex approaches to the treatment of those diseases.

Depression is common in patients with diabetes mellitus [2]. The prevalence of any depression (major or minor forms) is significantly higher in patients with type 2 diabetes mellitus (T2DM) than in those without diabetes (17.6 vs. $9.8 \%$ ) [3]. Depression has been shown to be associated to treatment non-adherence [4].

\section{Diabetes, Obesity and Depression}

In the development of T2DM obesity seems to play a major role. In a study on more than 9,000 adolescents, depression was able to induce obesity, whereas obesity did not increase the risk of depression [5]. In a meta-analysis in adults depression was linked to the manifestation of T2DM [6]. Thus depression seems to be a major driving force at the beginning of the disease. In patients with T2DM daily negative moods adversely affect blood glucose control [7]. Interestingly, depression seems to also influence markers of inflammation [8] which in turn could be responsible for the consecutive manifestation of diabetes or deterioration of blood glucose control in overt diabetes. The relation between obesity and depression has previously been described the other way round, too [9]. As $80 \%$ of diabetic patients are obese or overweight, overweight may play also a role in the development of comorbid depression [10]. Additionally, overweight might play a role in the treatment adherence with insulin or other weightincreasing agents as patients fear weight gain [11]. Recently it has been shown that depressive symptoms do not necessarily result in poor glycemic control. On the other hand patients with T2DM and poor glycemic control are at moderate risk for worsening of depressive symptoms [12].

\section{Consequences of Depression}

The pathophysiologic relationship between comorbid depression and diabetes is poorly understood. As with other severe chronic diseases, psychological factors associated with the hardship of diabetes (diabetes distress) may trigger or enhance depressive symptoms [13]. Diabetes distress is a condition distinct from depression that is linked to diabetes outcomes. In an 18month study the odds ratio for becoming depressed was higher for those being female, previously depressed, or experiencing more chronic stress [14]. Depression seems to affect a number of important steps in patient management, including self-care control problems, less likelihood to report positive attitude, self-care ability and self-care adherence. Depressed patients were less likely to report perceived control of diabetes despite a comparable level of knowledge, self-care understanding, and perceived importance of self-care [15]. Table 1 gives typical characteristics of patients with diabetes and depression [16].

The question is: Do they not want or are they not able to comply?

As a consequence the comorbidity of diabetes and depression is associated with diabetes complications [17] and a significantly increased risk of death, beyond that due to having either diabetes or depression alone [18].

The challenge therefore seems to be to improve depression with an impact on patient management and with a possible impact on long-term medical cost.

\section{Behavioral Treatment}

A recent elegant, randomized controlled trial investigated the possible effects of cognitive behavioral therapy (CBT) and

\section{KARGER}

Fax +497614520714

Information@Karger.de

www.karger.com (c) 2009 S. Karger GmbH, Freiburg

Accessible online at:

www.karger.com/ofa
Univ. Prof. Dr. med. Hermann Toplak

Department of Medicine

Medical University Graz

Auenbrugger Platz 15, 8036 Graz, Austria

Tel. +43 316 385-80246, Fax -3812

hermann.toplak@medunigraz.at 
Table 1. Characteristics of patients with diabetes and depression [16]

Exhibit unfavorable blood glucose control

Adhere less to therapeutic medical recommendations

Drop out of weight reduction programs more frequently

Are more likely to smoke

Induce higher costs

blood glucose awareness training (BGAT) in type 1 diabetes mellitus (T1DM). Interestingly, CBT could effectively help T1DM patients with comorbid depression [19].

To our knowledge no recent studies on CBT in T2DM and depression are available. In T2DM without diagnosis of depression behavioral modification therapy has also shown positive effects though to a lesser extent than in T1DM [20].

\section{Drug Treatment}

Data on the impact of antidepressant therapy on metabolic and anthropometric measures in diabetic patients with comorbid depression are still scarce and have primarily focused on fluoxetine and sertraline, both with similar effects on depression and blood glucose control [21, 22]. Additionallly, a comparison of fluoxetine and paroxetine has been published, with a positive trend for fluoxetine concerning HbA1c [23]. Another study evaluated bupropion and revealed that sustained improvement in mood and BMI led to an improvement in HbA1c [24]. S-citalopram has been evaluated for diabetes mellitus, too; it improved symptoms of depression but did not significantly alter factors of glucose control [25]. We have recently published data on milnacipran [26], a serotonin norepinephrine reuptake inhibitor, which has been shown to reduce binge eating-like behavior [27]. This kind of eating disorder is common but underreported in patients with the metabolic syndrome and diabetes mellitus. Our data show that treatment with milnacipran improves depression, weight, and Hba1c in T2DM. The crucial event seems to be the reduction in depression (Beck Depression Inventory, fig. 1) with consecutive effects on weight (fig. 2) and HbA1c (fig. 3). The drawbacks are that the study was not randomized and double-blind, but as the beneficial effects were only present in responders to antidepressive treatment, the information seems to be valid, especially as the concomitant treatment was similar in both groups. Further studies are needed to investigate such effects.

It has to be stressed that medical students and later on general practitioners hold negative attitudes towards patients with psychiatric disorders [28]. In the future it has to be ensured that students and doctors develop an empathetic and positive approach to mental health patients and their treatment.

\section{Conclusions}

Stress, diabetes distress, and depression are likely to influence patients' treatment adherence and compliance. Patient educa-

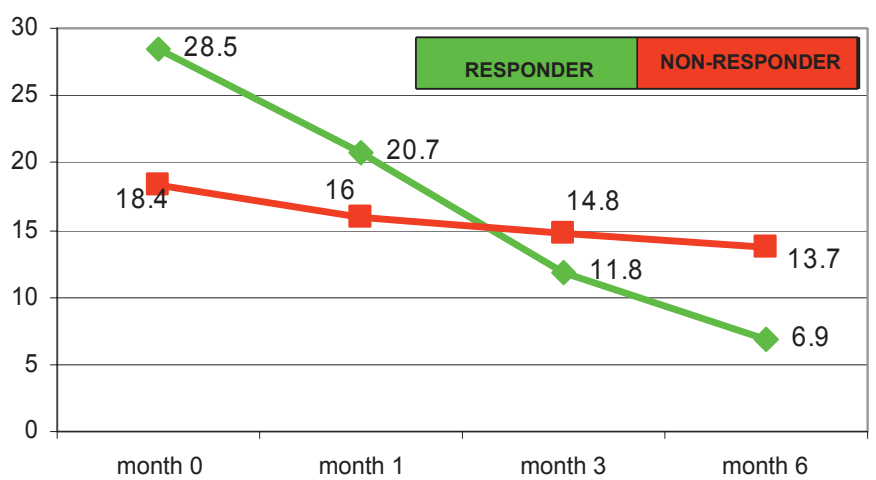

Fig. 1. Effect of milnacipran on Beck Depression Inventory (mean values) [26].

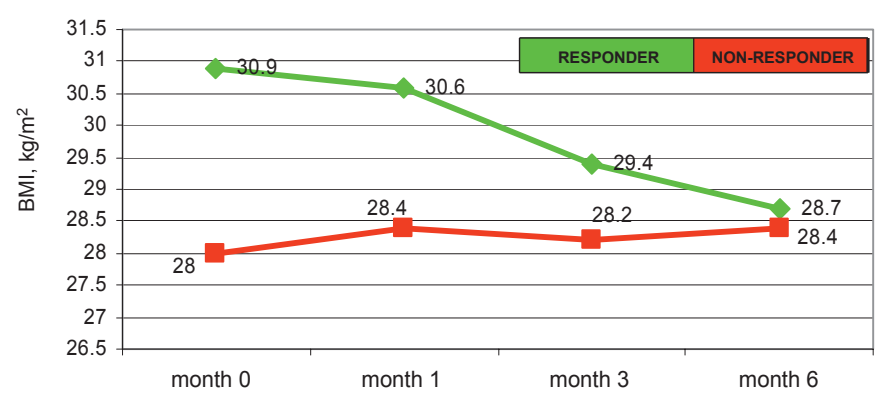

Fig. 2. Effect of milnacipran on BMI (median values) [26].

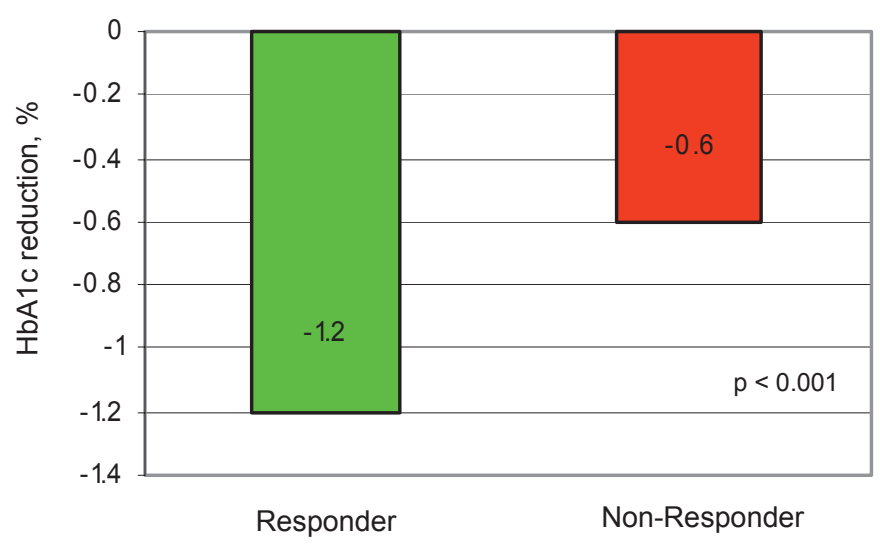

Fig. 3. Effect of milnacipran on HbA1c. Mean reduction: baseline $\rightarrow$ month 6 [26].

tion alone does not seem to be sufficient to overcome these obstacles. Behavior therapy and, if necessary, drug treatment provide valuable tools in a multifactorial, more holistic approach to patient management. There is substantial need for practicable tools to detect underlying psychiatric diseases in everyday practice. Additionally, exchange and cooperation between psychiatrists and psychologists on the one side and internists, diabetologists as well as general practitioners on the other side could lead to improved health care in chronic diseases such as diabetes mellitus. 


\section{References}

1 Kapusta ND, Plener PL, Schmid R, Thau K, Walter H, Lesch OM: Multiple substance use among young males. Pharmacol Biochem Behav 2007;86(2):306311.

2 Katon WJ: The comorbidity of diabetes mellitus and depression: Am J Med 2008;121(11 suppl 2): S8-S15.

3 Ali S, Stone MA, Peters JL, Davies MJ, Khunti K: The prevalence of co-morbid depression in adults with type 2 diabetes: a systematic review and metaanalysis. Diabet Med 2006;23(11):1165-1173.

$\checkmark 4$ Gonzalez JS, Peyrot M, McCarl LA, Collins EM, Serpa L, Mimiaga MJ, Safren SA: Depression and diabetes treatment nonadherence: a meta-analysis. Diabetes Care 2008;31(12):2398-2403.

$\checkmark 5$ Goodman E, Whitaker RC: A prospective study of the role of depression in the development and persistence of adolescent obesity. Pediatrics 2002;110: 497-504.

6 Knol MJ, Twisk JW, Beekman AT, Heine RJ, Snoek FJ, Pouwer F: Depression as a risk factor for the onset of type 2 diabetes mellitus. A meta-analysis. Diabetologia 2006;49(5):837-45.

7 Skaff MM, Mullan JT, Almeida DM, Hoffman L, Masharani U, Mohr D, Fisher L: Daily negative mood affects fasting glucose in type 2 diabetes. Health Psychol 2009;28(3):265-272.

$\checkmark 8$ Dixon JB, Hayden MJ, Lambert GW, Dawood T, Anderson ML, Dixon ME, O'Brien PE: Raised CRP levels in obese patients: symptoms of depression have an independent positive association. Obesity 2008;16(9):2010-2015.

$\checkmark 9$ Dong C, Sanchez LE, Price RA: Relationship of obesity to depression: a family-based study. Int J Obes Relat Metab Disord 2004;28(6):790-795.

10 Onyike CU, Crum RM, Lee HB, Lyketsos CG, Eaton WW: Is obesity associated with major depression? Results from the Third National Health and Nutrition Examination Survey. Am J Epidemiol 2003;158(12):1139-1147.

11 Russell-Jones D, Khan R. Insulin-associated weight gain in diabetes - causes, effects and coping strategies. Diabetes Obes Metab 2007;9(6):799-812.
Aikens JE, Perkins DW, Lipton B, Piette JD: Longitudinal analysis of depressive symptoms and glycemic control in type 2 diabetes. Diabetes Care 2009;32(7):1177-1181.

13 Talbot F, Nouwen A: A review of the relationship between depression and diabetes in adults: is there a link ? Diabetes Care 2000;23:1556-1562.

14 Fisher L, Mullan JT, Skaff MM, Glasgow RE, Arean P, Hessler D: Predicting diabetes distress in patients with type 2 diabetes: a longitudinal study. Diabet Med 2009;26(6):622-627.

15 Egede LE, Ellis C: The effects of depression on diabetes knowledge, diabetes self-management, and perceived control in indigent patients with type 2 diabetes. Diabetes Technol Ther 2008;10(3):213-219.

16 Albus C, Kulzer B, Petrak F, Kurse J, Hirsch A, Herpertz S: Psychosoziales und Diabetes mellitus. Praxis-Leitlinie der Deutschen Diabetes Gesellschaft (DDG). Diabetes Stoffwechsel 2004;13(suppl 2): 77-83.

17 de Groot M, Anderson R, Freedland KE, Clouse RE, Lustman PJ: Association of depression and diabetes complications: a meta-analysis. Psychosom Med 2001;63(4):619-630.

18 Egede LE, Nietert PJ, Zheng D: Depression and all-cause and coronary heart disease mortality among adults with and without diabetes. Diabetes Care 2005;28(6):1339-1345.

19 Snoek FJ, van der Ven NC, Twisk JW, Hogenelst $\mathrm{MH}$, Tromp-Wever AM, van der Ploeg HM, Heine RJ: Cognitive behavioural therapy (CBT) compared with blood glucose awareness training (BGAT) in poorly controlled type 1 diabetic patients: long-term effects on HbA moderated by depression. A randomized controlled trial. Diabet Med 2008;25(11):1337-1342.

20 Forlani G, Lorusso C, Moscatiello S, Ridolfi V, Melchionda N, Di Domizio S, Marchesini G: Are behavioural approaches feasible and effective in the treatment of type 2 diabetes? A propensity score analysis vs. prescriptive diet. Nutr Metab Cardiovasc Dis 2009;19(5):313-320.
1 Goodnick PJ: Use of antidepressants in treatment of comorbid diabetes mellitus and depression as well as in diabetic neuropathy. Ann Clin Psychiatry 2001;13(1):13-41.

22 Lustman PJ, Clouse RE, Nix BD, Freedland KE, Rubin EH, McGill JB, Williams MM, Gelenberg AJ, Ciechanowski PS, Hirsch IB: Sertraline for prevention of depression recurrence in diabetes mellitus: a randomized, double-blind, placebo-controlled trial. Arch Gen Psychiatry 2006;63(5):521-529.

23 Gülseren L, Gülseren S, Hekimsoy Z, Mete L: Comparison of fluoxetine and paroxetine in type II diabetes mellitus patients. Arch Med Res 2005; 36(2):159-165.

24 Lustman PJ, Williams MM, Sayuk GS, Nix BD, Clouse RE: Factors influencing glycemic control in type 2 diabetes during acute- and maintenancephase treatment of major depressive disorder with bupropion. Diabetes Care 2007;30(3):459-466.

25 Amsterdam JD, Shults J, Rutherford N, Schwartz S: Safety and efficacy of s-citalopram in patients with co-morbid major depression and diabetes mellitus. Neuropsychobiology 2006;54(4):208-214.

26 Abrahamian H, Hofmann P, Prager R, Toplak $\mathrm{H}$ : Diabetes mellitus and co-morbid depression: treatment with milnacipran results in significant improvement in both diseases (results from the Austrian MDDM study group). Neuropsychiatr Dis Treatm 2009;5:261-266.

27 El-Giamal N, de Zwaan M, Bailer U, Strnad A, Schüssler P, Kasper S: Milnacipran in the treatment of bulimia nervosa: a report of 16 cases. Eur Neuropsychopharmacol 2003;13(2):73-79.

28 Dixon RP, Roberts LM, Lawrie S, Jones LA, Humphreys MS: Medical students' attitudes to psychiatric illness in primary care. Med Educ 2008;42(11): 1080-1087.

\section{On the Contents of This Issue}

Single studies have indicated that after weight loss weight regain is promoted by continuously disproportionately lowered leptin levels. Holm (Denmark) et al. [1] measured serum leptin levels of 115 children at baseline upon entry into a 12-week-weight loss program. 90 children completed the program, and 68 children were followed up for 28 months. Leptin levels were measured on day 82 and after 10, 16 and 28 months. The investigators found no evidence for the hypothesis that disproportionately low concentrations of leptin contribute to a later weight gain. Instead, leptin increases mirrored increases in BMI-SDS during weight regain and the leptin-BMI SDS relationship seen during follow-up resembled the baseline leptin-BMI-SDS relationship.
In a second pediatric study, de Toia (Germany) et al. [2] examined the relationship between motor abilities and body weight in 1,228 kindergarten children. In school-aged overweight and obese children motor abilities have been shown to be reduced; however, little is known about preschoolers. A total of $13.1 \%$ of the young children qualified as overweight or obese as based on German reference data. The investigators used the modified Karlsruhe Motor Ability Screening Test 3-6 (KMS 3-6) to determine speed, strength, muscular endurance, coordination, and flexibility; the reliability of all test items has been shown to be high ( $r>0.8)$. Children who had motor abilities in the lowest two quintiles of the reference range were regarded as having below-average motor abilities. Below-average motor abilities were found in $44.0-47.3 \%$ of all children, the highest percentage applied to the one-leg-stand test. No differenc- 
es were detected between normal weight and overweight/ obese children. These results are largely in accordance with the few previous studies focusing on pre-schoolers. The authors advocate preventive measurements to avoid both overweight and motor deficits. It appears that overweight and obesity lead to more physical inactivity in older children only, thus also explaining the poor motor abilities found beyond the pre-school age range.

Population-based data indicate that mood and anxiety disorders occur more commonly in obese individuals. Prior to such more recent epidemiological studies an elevated rate of these disorders had repeatedly been detected in patients seeking weight loss. Legenbauer (Germany) et al. [3] address the important question as to whether or not depression and anxiety predict weight outcomes in participants of a conventional weight loss treatment program, obesity surgery patients, and obese control individuals. The authors used a structured psychiatric interview to diagnose mood and anxiety disorders as well as disturbed eating patterns. The conventional treatment group encompassed almost twice as many participants as the control group (240 vs. 128); the surgery group included 151 patients. Stepwise linear regression analysis with presence of comorbid depressive and/or anxiety disorder, presence of any mental disorder, and number of comorbid disorders as independent variables revealed that the presence or absence of a current comorbid depressive and/or anxiety disorder had a statistically significant impact on weight loss of the bariatric patients and the controls, but not of the obese subjects receiving conventional treatment. Bariatric patients with a comorbid mood and/or anxiety disorder lost significantly less weight compared with those bariatric patients without any current mental disorder at baseline. Similarly, a current mood and/or anxiety disorder at baseline predicted a poor weight outcome in the obese controls. In contrast, no effect of psychiatric disorders on the outcome of conventionally treated patients was observed. Because the number of obese subjects with a comorbid psychiatric disorder was relatively small, both - mood and anxiety disorder - were co-analyzed. The authors recommend larger studies to disentangle the effects of each of these disorders. The authors also recommend further studies to identify psychopathological factors predisposing to poor treatment outcomes; such knowledge should prove helpful in the optimization of interventions.

Obesity is commonly attributed to factors exerting a direct impact on energy intake or expenditure. Accordingly, conventional therapy and prevention strategies focus on reduction of caloric intake and on an increase of physical activity. Chaput and Tremblay (Canada) [4] convincingly argue that this reductionistic approach based on energy balance has its limitations and conceivably even obscures alternative strategies for tackling the obesity epidemic. The authors base their argument on two phenomena, which characterize our modern lifestyle: i) Sleep loss is an endemic condition of modern societies; several studies have shown that reduced sleep duration is associated with excess body weight. Longitudinal studies have shown that short sleeping hours in children predict overweight or obesity to a greater extent than low physical activity levels and television viewing. The positive relation between a short sleep duration and excess body weight is underpinned by findings in sleep-deprived individuals revealing an increase in the orexigenic hormone ghrelin and a decrease in the anorexigenic hormone leptin. Based on such data and the fact that sleep is probably the most sedentary of all activities, it becomes evident that an increase in the time allocated to this low calorie burning activity should not be negatively perceived given the beneficial impact of sleep on the regulation of energy balance. ii) The second factor discussed by Chaput and Tremblay is the recent increase in mental activity. Globalization and technological demands have favored a progressive switch from physically demanding tasks to knowledge-based work (KBW). Because brain activity depends on glucose metabolism, the authors argue that KBW leads to an increased energy intake. Indeed, some studies have shown that KBW increases plasma glucose instability and induces overeating. The authors conclude by pointing out that 'sedentariness' is commonly solely viewed in terms of its direct impact on energy expenditure. The net impact of these low calorie burning activities on energy balance has, however, been widely disregarded. Sleep and KBW are both subsumed under 'sedentariness' that may actually have opposite effects on energy intake.

Singmann (Germany) et al. [5] looked at a gene-gene interaction based on variants of the apolipoprotein A5 (APOA5) gene and of the upstream stimulatory factor 1 gene (USF1), which is one of the transcription factors of APOA5. Based on previous findings, the investigators sought to identify an interaction between these two genes on the risk for developing the metabolic syndrome in 1,622 individuals aged 54-74 years who were ascertained within the German population-based KORA survey 4. Seven APOA 5 SNPs were analyzed in combination with six USF1 SNPs applying logistic regression in an additive model adjusting for age and sex. The metabolic syndrome was defined according to the National Cholesterol Education Program's Adult Treatment Panel III. 41\% of the subjects fulfilled the criteria for the metabolic syndrome. Whereas two SNP combinations revealed a nominal gene-gene interaction, these associations did no remain significant after correction for multiple testing.

El-Gilany (Egypt) and El-Wehady [6] analyzed the prevalence of obesity in a Saudi obstetric population. The 
authors undertook their study in light of the known association of obesity and a higher risk of complications during pregnancy and more frequent health problems in the offspring. Because a recent study revealed that over one half of Saudi nonpregnant women of childbearing age were overweight or obese, the investigators set out to determine rates of obesity among pregnant women in the Kingdom of Saudi Arabia. The study was based on all women presenting for antenatal care in the 47 primary healthcare centers in Al-Hassa, the largest province in Saudi Arabia's Eastern region with a population of about $900,000.52 .2 \%$ of the pregnant women were overweight or obese, including $4.7 \%$ with extreme obesity. Obesity rates were higher among urban women, among those with satisfactory family income, in older age groups, and among those of higher parity. Logistic regression revealed that the most important significant independent predictors of obesity were parity of four and more, urban residence, and satisfactory family income. The prevalence rate of obesity at this early stage of pregnancy may actually have been underestimated because women with prepregnancy diabetes, hypertension, and other chronic diseases known to be associated with higher BMI and the metabolic syndrome had been excluded.

\section{References}

1 Holm J-C, Gamborg M, Ward L, Ibsen KK, Gammeltoft S, Sørensen TIA Heitmann BL: Longitudinal analysis of leptin variation during weight regain after weight loss in obese children. Obes Facts 2009;2(4):243-248.

2 de Toia D, Klein D, Weber S, Wessely N, Koch B, Tokarski W, Dordel S, Strüder H, Graf C: Relationship between anthropometry and motor skills at pre-school age. Obes Facts 2009;2(4):221-225.

3 Legenbauer T, de Zwaan M, Benecke A, Mühlhans B, Petrak F, Herpertz S: Depression and anxiety: their predictive function for weight loss in obese individuals. Obes Facts 2009;2(4):227-234.

4 Chaput JP, Tremblay A: Obesity and physical inactivity: the relevance of reconsidering the notion of sedentariness. Obes Facts 2009;2(4):249-254.

5 Singmann P, Baumert J, Herder C, Meisinger C, Holzapfel C, Klopp N, Wichmann H-E, Klingenspor M, Rathmann W: Gene-gene interaction between APOA5 and USF1: two candidate genes for the metabolic syndrome. Obes Facts 2009;2(4):235-242.

6 El-Gilany AH, El-Wehady A: Prevalence of obesity in a Saudi obstetric population. Obes Facts 2009;2(4):217-220. 